\title{
An exponential lower bound for a constraint propagation proof system based on ordered binary decision diagrams preliminary version
}

\author{
Jan Krajíček*† \\ Academy of Sciences and Charles University, Prague
}

\begin{abstract}
We prove an exponential lower bound on the size of proofs in the proof system operating with ordered binary decision diagrams introduced by Atserias, Kolaitis and Vardi [2]. We utilize (somewhat indirectly) feasible interpolation.
\end{abstract}

Atserias, Kolaitis and Vardi [2] generalized refutation proof systems from Boolean logic to the realm of Constraint Satisfaction Problems (CSP), viewing it as a special case of constraint propagation. This brings constraint propagation within the reach of proof complexity methods and, on the other hand, introduces a new class of propositional proof systems (pps) in the sense of Cook and Reckhow [6].

In the Boolean case Atserias et.al. [2] introduced and studied a particular pps operating with ordered binary decision diagrams (OBDD), and they obtained a number of proof complexity results about it. In particular, they compared the pps with several well-known pps', including resolution, constant-depth Frege systems and small-coefficients cutting planes.

A problem left open in Atserias et.al.[2] is to prove a lower bound for this new pps, although they have obtained an interesting partial result: a feasible interpolation theorem (also monotone, and hence a lower bound too) for refutations using OBDDs with certain specific orders of variables ([2, Thm.9]). In this paper we prove an exponential lower bound without any restrictions. We deduce first, as a straightforward corollary of the feasible interpolation theorem for semantic derivations from Krajíček [10], a feasible interpolation theorem for refutations with OBDDs for special orders of variables. Then we show how

\footnotetext{
${ }^{*}$ Keywords: proof complexity, OBDD, constraint propagation, feasible interpolation.

†Supported in part by grants A1019401, AV0Z10190503, MSM0021620839, 201/05/0124, and LC505.
} 
to deduce even from such a restricted interpolation a lower bound for OBDD refutations satisfying no apriori restrictions ${ }^{1}$.

The paper is organized as follows. The OBDDs and the new proof system of Atserias et.a.[2] are recalled in Section 1. Section 2 recalls the feasible interpolation theorem for semantic derivations of Krajíček [10]. The lower bound is proved in Section 3.

We do not review basics of proof complexity or constraint propagation. The reader my consult $[9,10,11]$ for the former and $[2,7]$ for the latter.

\section{The OBDD proof system}

Proofs in a lot of usual pps' are organized into proof lines, the lines being syntactic objects representing Boolean functions. For example, in resolution the lines are clauses, in cutting planes these are integer inequalities, and in Frege systems these are arbitrary propositional formulas.

The lines in the proof system of Atserias et.al.[2] are OBDDs. OBDDs are particular branching programs (BP). A BP is a directed acyclic graph with one root which has no incoming edges and exactly two outgoing edges, with two sinks (nodes with no outgoing edges) labeled by 0 and 1 respectively, and with all other nodes (inner nodes) having exactly two outgoing edges and any number of incoming edges. Every node except the sinks is labeled by one of the variables $x_{i}$ and the two edges leaving the node are labeled $x_{i}=0$ and $x_{i}=1$, respectively. A truth assignment to variables thus determines a unique path from the root to a sink. In this way a BP defines a Boolean function, sending the assignment to the value labeling the sink the path ends in. Every Boolean function can be represented by a BP and, in fact, the minimal size of such a BP is tightly linked with the space complexity of the function, cf. Wegener [17].

$\mathrm{An} \mathrm{OBDD}$ is a $\mathrm{BP}$ in which variables are queried on every path at most once and in an order consistent with one specific linear ordering $\pi$ of all variables; an OBDD consistent with $\pi$ is called a $\pi$-OBDD. This class of BPs has been introduced by Bryant[4]. The main feature of OBDDs is that every Boolean function can be represented by a unique OBDD in a reduced form, and the algorithm transforming any OBDD into its reduced form is p-time. We shall not repeat the reduction and the arguments here (they are straightforward). As a consequence of this fact one can decide in p-time whether two OBDDs represent the same function and, in fact, decide various other relations between functions or perform various manipulations with them, also in p-time. In particular, one can decide in p-time whether a function defined by one OBDD majorizes a function defined by another OBDD. This makes OBDDs very useful data structure representations with applications in verification, model checking, computer-aided design of VLSI circuits, and other areas. See Bryant[5] or Wegener[18].

\footnotetext{
${ }^{1}$ We could have used the interpolation theorem of Atserias et.al.[2]; however, we give our own feasible interpolation theorem because it is a simple corollary of the general interpolation for semantic derivations and because we want that our presentation is complete (the proof of the interpolation theorem from [2] is due to appear in the full paper only).
} 
Atserias et.al.[2, Def.1] define a fairly general concept of CSP Refutations. This is a semantic concept, not a proof system in the sense of Cook and Reckhow [6]. It operates with constraints. A constraint is a pair consisting of a tuple of variables (not necessary all of them) and a relation on the set of all possible values of these variables. There are four inference rules: (1) initial constraints, (2) the join of two constraints which is simply the intersection of the two relations extended naturally to all variables occurring in either one of them, (3) the projection of a constraint which is the existential quantification, and (4) the weakening which allows to relax the constraint by enlarging its relation.

Atserias et.al.[2] studied in detail CSP refutations for Boolean logic and with constraints represented by OBDDs. The lower bound we prove is independent of any particular choice of inference rules such as above, as long as they are binary (this is inessential) and sound. Hence rather than defining the OBDD system with the four rules above, we define a "semantic" version of it. We shall call it the $O B D D$ proof system (Atserias et.al.[2] do not use any particular name).

For a OBDD $P$ in $n$ variables let $f_{P}$ denote the Boolean function with domain $\{0,1\}^{n}$ defined by $P$. For two Boolean functions $f$ and $g$, the symbol $f \geq g$ denotes that $f$ majorizes $g$ at every input. Note that a clause over $n$ variables is definable by an OBDD (under any order of variables) of size at most $2+n$.

Definition 1.1 Let $\mathcal{C}=\left\{C_{1}, \ldots, C_{m}\right\}$ be a set of clauses in variables $x=$ $\left(x_{1}, \ldots, x_{n}\right)$. Let $\pi$ be a linear ordering of variables $x$. A $\pi$-OBDD refutation of $\mathcal{C}$ is a sequence

$$
P_{1}, \ldots, P_{k}
$$

of $\pi-O B D D$ s such that $f_{P_{k}} \equiv 0$, and every $P_{i}$ is either a $\pi-O B D D$ representing a Boolean function defined by a clause from $\mathcal{C}$, or:

$$
f_{P_{i}} \geq f_{P_{j_{1}}} \wedge f_{P_{j_{2}}}
$$

for some $j_{1}, j_{2}<i$. An OBDD refutation is a $\pi$-OBDD refutation, for any linear ordering $\pi$ of variables.

The size of the refutation is the sum of the sizes of $P_{i}$ ', the number of steps (sometimes called also the length) is the $k$.

The following lemma is a consequence of the above remarks on the uniqueness of OBDDs.

Lemma 1.2 The OBDD refutations are sound and complete, and form a proof system in the sense of Cook and Reckhow [6].

Atserias et.al.[2] showed that OBDD refutations p-simulate resolution but have an exponential speed-up over it, p-simulate cutting planes with small coefficients $C P^{*}$, and are incomparable with constant depth Frege systems. 


\section{Feasible interpolation}

Let $A(x, y)$ and $B(x, z)$ be two propositional formulas having the indicated occurrences of variables from tuples $x=\left(x_{1}, \ldots, x_{n}\right), y=\left(y_{1}, \ldots, y_{s}\right)$ and $z=$ $\left(z_{1}, \ldots, z_{t}\right)$. Propositional interpolation theorem says that if $A \wedge B$ is unsatisfiable then there is a formula $I(x)$, an interpolant $A$ and $B$, such that both $A \wedge I$ and $B \wedge \neg I$ are unsatisfiable.

The idea of feasible interpolation ${ }^{2}$ is simple: For a given proof system $\mathbf{P}$ establish an upper bound on the computational complexity of an interpolant of $A$ and $B$ in terms of the size of a $\mathbf{P}$-proof of the unsatisfiability of $A \wedge B$. Then any pair $A$ and $B$ which is hard to interpolate yields formula $A \wedge B$ that must have large $\mathbf{P}$-proofs of the unsatisfiability. Unconditional lower bounds are obtained by considering a monotone version of the above idea (see below).

In our lower bound proof we apply the general theorem on feasible interpolation for semantic derivations from Krajíček[10]. We now recall the necessary definitions and facts fromthat paper. We assume the reader is familiar with the concept of communication complexity of a function, with inputs distributed among two players: It is the minimal number of bits they need to communicate in the worst case (i.e. the maximum over all inputs) in order to compute the function. See Kushilevitz and Nisan[13] for details.

Let $N=n+s+t$ be natural numbers fixed for the rest of the section. The semantic rule allows to infer a subset $C \subseteq\{0,1\}^{N}$ from two subsets $A, B \subseteq$ $\{0,1\}^{N}$ iff $C \supseteq A \cap B$. A semantic refutation of the sets $A_{1}, \ldots, A_{m} \subseteq\{0,1\}^{\bar{N}}$ is a sequence of sets $B_{1}, \ldots, B_{k} \subseteq\{0,1\}^{N}$ such that $B_{k}=\emptyset$, each $B_{i}$ is either one of $A_{j}$ or derived from two previous $B_{i_{1}}, B_{i_{2}}$ by the semantic rule.

Let $A \subseteq\{0,1\}^{N}$ and assume $u, v \in\{0,1\}^{n}, y^{u} \in\{0,1\}^{s}$ and $z^{v} \in\{0,1\}^{t}$. Consider three tasks:

1. Decide whether $\left(u, y^{u}, z^{v}\right) \in A$.

2. Decide whether $\left(v, y^{u}, z^{v}\right) \in A$.

3. If $\left(u, y^{u}, z^{v}\right) \in A \not \equiv\left(v, y^{u}, z^{v}\right) \in A$ find $i \leq n$ such that $u_{i} \neq v_{i}$.

These tasks can be solved by two players, one knowing $u, y^{u}$ (the U-player) and the other one knowing $v, z^{v}$ (the V-player). The communication complexity of $A, C C(A)$, is the minimal number of bits they need to exchange in the worst case in solving any of these three tasks ${ }^{3}$.

Consider one more task:

\footnotetext{
${ }^{2}$ First formulated in Krajíček[8](circulating in 1991), and subsequently (but apparently independently in some cases) used for a variety of proof complexity results (new lower bounds, independence results for bounded arithmetic, establishing links between proof complexity and cryptography, automatizability of proof search, etc.) in Razborov[16], Bonet, Pitassi and Raz[3], Krajíček[10], Krajíček and Pudlák[12], Pudlák[14], and a number of other papers; see $[11,15]$ for overviews.

${ }^{3}$ We tacitly assume that the decomposition $N=n+s+t$, and hence the distribution of input bits to the players, is apriori determined.
} 
4. If $\left(u, y^{u}, z^{v}\right) \in A$ and $\left(v, y^{u}, z^{v}\right) \notin A$ either find $i \leq n$ such that

$$
u_{i}=1 \wedge v_{i}=0
$$

or learn that there is some $u^{\prime}$ satisfying

$$
u^{\prime} \geq u \wedge\left(u^{\prime}, y^{u}, z^{v}\right) \notin A
$$

( $u \leq u^{\prime}$ means $\bigwedge_{i \leq n} u_{i} \leq u_{i}^{\prime}$; the players are not required to find any $u^{\prime}$.)

The monotone communication complexity w.r.t. $U$ of $A, M C C_{U}(A)$, is the minimal $r \geq C C(A)$ such that the task 4 . can be solved communicating $\leq r$ bits in the worst case.

For $A \subseteq\{0,1\}^{n+s}$ define the set $\tilde{A}$ by:

$$
\tilde{A}:=\bigcup_{(a, b) \in A}\left\{(a, b, c) \mid c \in\{0,1\}^{t}\right\}
$$

where $a, b, c$ range over $\{0,1\}^{n},\{0,1\}^{s}$ and $\{0,1\}^{t}$ respectively, and similarly for $B \subseteq\{0,1\}^{n+t}$ define $\tilde{B}$ :

$$
\tilde{B}:=\bigcup_{(a, c) \in B}\left\{(a, b, c) \mid b \in\{0,1\}^{s}\right\} .
$$

Theorem 2.1 (Krajíček[10]) Let $A_{1}, \ldots, A_{m} \subseteq\{0,1\}^{n+s}$ and $B_{1}, \ldots, B_{\ell} \subseteq$ $\{0,1\}^{n+t}$. Assume that there is a semantic refutation $D_{1}, \ldots, D_{k}$ of the sets $\tilde{A}_{1}, \ldots, \tilde{A}_{m}, \tilde{B}_{1}, \ldots, \tilde{B}_{\ell}$ such that $C C\left(D_{i}\right) \leq r$ for all $i \leq k$.

Then the two sets

$$
U=\left\{u \in\{0,1\}^{n} \mid \exists y^{u} \in\{0,1\}^{s} ;\left(u, y^{u}\right) \in \bigcap_{j \leq m} A_{j}\right\}
$$

and

$$
V=\left\{v \in\{0,1\}^{n} \mid \exists z^{v} \in\{0,1\}^{t} ;\left(v, z^{v}\right) \in \bigcap_{j \leq \ell} B_{j}\right\}
$$

can be separated by a circuit of size at most $(k+2 n) 2^{O(r)}$.

Moreover, if the sets $A_{1}, \ldots, A_{m}$ satisfy the following monotonicity condition w.r.t. $U$ :

$$
\left(u, y^{u}\right) \in \bigcap_{j \leq m} A_{j} \wedge u \leq u^{\prime} \rightarrow\left(u^{\prime}, y^{u}\right) \in \bigcap_{j \leq m} A_{j}
$$

and $M C C_{U}\left(D_{i}\right) \leq t$ for all $i \leq k$, then there is even a monotone circuit separating $U$ from $V$ of size at most $(k+n) 2^{O(r)}$. 


\section{The lower bound}

Assume $N=n+s+t$, and let $x, y$ and $z$ be disjoint tuples of $n, s$ and $t$ variables, as in the previous section. Let $V a r$ be the set of all these variables in $x, y, z$. A linear ordering $\pi$ of $\operatorname{Var}$ is block consistent with $y<z<x$ iff $\pi$ puts all $y$-variables before all $z$ - and $x$-variables, and all $x$-variables after all $z$-variables.

Lemma 3.1 Let $\pi$ be a linear ordering of Var that is block consistent with $y<z<x$. Assume a subset $A \subseteq\{0,1\}^{N}$ is definable by a $\pi-O B D D P$ of size $S$. Then both $C C(A)$ and $M C C_{U}(A)$ are bounded above by $O(\log (S) \cdot \log (n))$.

\section{Proof :}

The U-player, knowing $y^{u}$, starts the path through $P$ and sends to the other player $\log (S)$ bits indicating the last node he reached, i.e. the first node not querying a $y$-variable. Then the $\mathrm{V}$-player, knowing $z^{v}$, continues in the path and sends to the U-player $\log (S)$ bits naming the node with first query of an $x$-variable he got to. Call this node $p$.

After this stage they both continue from $p$ the computation individually, using $u$ and $v$ respectively, and send each other 1 bit - their output. If the outputs differ then they use binary search looking for a node whose $x$-query they answered differently. This involves at $\operatorname{most} \log (n)$ steps, each time sending each other $\log (S)$ bits naming the node querying the particular $x$-variable their paths got to. Hence the total estimate to $C C(A)$ is $O(\log (S) \cdot \log (n))$.

For the monotone complexity assume $\left(u, y^{u}, z^{v}\right) \in A$ while $\left(v, y^{u}, z^{v}\right) \notin A$. Let $p_{1}:=p, \ldots, p_{\ell}$ be the path from $p$ determined by $v$ (call it the $v$-path), with $p_{\ell}$ being the sink labeled by 0 . In particular, $\ell \leq n$.

The U-player indicates by sending 1 bit whether or not there is a $u^{\prime} \geq u$ such that $\left(u^{\prime}, y^{u}, z^{v}\right) \notin A$, i.e. the $u^{\prime}$-path from $p$ leads to $p_{\ell}$. If so, the players stop, having answered the 4 . task.

Otherwise the $\mathrm{V}$-player picks a point in the middle of his path, say $p_{\ell / 2}$, and sends its name $(\log (S)$ bits) to the U-player. The U-player sends back 1 bit indicating whether or not there is $u^{\prime} \geq u$ such that the $u^{\prime}$-path from $p$ leads to $p_{\ell / 2}$.

If there is such a $u^{\prime}$ they will move to sub-path $p_{\ell / 2}, \ldots, p_{\ell}$, otherwise to the sub-path $p_{1}, \ldots, p_{\ell / 2}$. Note that the U-player does not know the whole $v$-path from $p$, only the endpoints of the current sub-path.

In general, after $r$ rounds of this process, the players have a sub-path of length $\leq n / 2^{r}$ (the U-player knows its endpoints) such that there is a $u^{\prime} \geq u$ for which the $u^{\prime}$-path from $p$ leads to the starting node of the sub-path but no such $u^{\prime} \geq u$ exists for the end-node of the sub-path. Hence in at most $\log (n)$ rounds, in which they have exchanged at $\operatorname{most} \log (n) \cdot(1+\log (S))$ bits, they

find a node $p_{w}$ on the $v$-path such that, in particular, for no $u^{\prime} \geq u$ does the $u^{\prime}$-path leads from $p_{w}$ to $p_{w+1}$. If $x_{i}$ is the label of $p_{w}$ this means (as $x_{i}$ is not querried at any earlier node) that $v_{i}=0$ while $u_{i}=1$, and the players have found what they wanted. 
q.e.d.

The following lemma is then an immediate consequence of Theorem 2.1 and Lemma 3.1 (we estimate the number of lines by the size). Atserias et.al.[2, Thm.9] give an estimate to the circuit complexity of an interpolant for orderings $\pi$ block consistent with $y<x<z$.

Lemma 3.2 Let $\pi$ be a linear ordering of Var that is block consistent with $y<z<x$. Let $A_{1}, \ldots, A_{m} \subseteq\{0,1\}^{n+s}$ and $B_{1}, \ldots, B_{\ell} \subseteq\{0,1\}^{n+t}$. Assume that there is a semantic $O B D \bar{D}$ refutation the sets $\tilde{A}_{1}, \ldots, \tilde{\tilde{A}}_{m}, \tilde{B}_{1}, \ldots, \tilde{B}_{\ell}$ of size $S$.

Then the two sets

$$
U=\left\{u \in\{0,1\}^{n} \mid \exists q^{u} \in\{0,1\}^{s} ;\left(u, y^{u}\right) \in \bigcap_{j \leq m} A_{j}\right\}
$$

and

$$
V=\left\{v \in\{0,1\}^{n} \mid \exists r^{v} \in\{0,1\}^{t} ;\left(v, z^{v}\right) \in \bigcap_{j \leq \ell} B_{j}\right\}
$$

can be separated by a circuit of size at most $S^{O(\log (n))}$.

Moreover, if the sets $A_{1}, \ldots, A_{m}$ satisfy the monotonicity condition from Theorem 2.1 then there is even a monotone circuit separating $U$ from $V$ of size at most $S^{O(\log (n))}$.

Let $A\left(x, y\right.$ and $B(x, z)$ be arbitrary formulas, where $x, y$ and $z$ are $n-, s^{-}$ and $t$-tuples of atoms respectively, as above, and $N=n+s+t$. We are going to construct a new formula $D_{A, B}(w, f)$, where $w$ is an $N$-tuple of variables $w_{i}$, and $f$ an $N^{2}$-tuple of variables $f_{i j}$ with $i, j \in[N]$.

Define first formulas $X_{j}$ by:

$$
\bigvee_{i \in[N]} w_{i} \wedge f_{i j}
$$

for $j \in[n]$, and similarly formulas $Y_{j}$ :

$$
\bigvee_{i \in[N]} w_{i} \wedge f_{i(n+j)}
$$

for $j \in[s]$, and $Z_{j}$ :

$$
\bigvee_{i \in[N]} w_{i} \wedge f_{i(n+s+j)}
$$

for $j \in[t]$.

Let $\operatorname{Map}(f)$ be the formula expressing that $f_{i j}$ defines a graph $\left\{(i, j) \mid f_{i j}=\right.$ $1\}$ of a permutation on $[N]$. Define formula $D_{A, B}(w, f)$ as:

$$
M a p(f) \wedge A(X, Y) \wedge B(X, Z) .
$$


Lemma 3.3 Let $\sigma$ be an arbitrary linear ordering of variables $w$ and $f$, and $\pi$ be an arbitrary linear ordering of variables $x, y$ and $z$.

Assume that formula $D_{A, B}(w, f)$ has a $\sigma-O B D D$ refutation of size $S$. Then formula $A(x, y) \wedge B(x, z)$ has a $\pi-O B D D$ refutation of size at most $S$.

\section{Proof :}

Assume $\sigma$ induces on variables $w$ ordering

$$
w_{i_{1}}<w_{i_{2}}<\ldots<w_{i_{N}}
$$

$\left\{i_{1}, \ldots, i_{N}\right\}=[N]$. Let $F$ be a permutation of $[N]$ such that $F\left(i_{r}\right)$ equals

- to $j$, if the $r$-th element of $\pi$ is $x_{j}$,

- to $n+j$, if the $r$-th element of $\pi$ is $y_{j}$,

- to $n+s+j$, if the $r$-th element of $\pi$ is $z_{j}$,

respectively.

Substitute in the whole refutation $f_{u v}:=1$ if $F(u)=v$, and $f_{u v}:=0$ otherwise. After the substitution each formula $X_{j}$ (and similarly $Y_{j}$ and $Z_{j}$ ) becomes equivalent to one of variables $w$ and the ordering of these $X, Y$ and $Z$ induced by the $\sigma$ ordering of these $w$ is isomorphic to the $\pi$ ordering of $x, y$ and $z$.

The substitution for variables $f$ satisfies $\operatorname{Map}(f)$. Hence the original $\sigma$ OBDD refutation becomes after the substitution a $\pi$-OBDD refutation of $A(x, y) \wedge$ $B(x, z)$.

\section{q.e.d.}

To prove the lower bound we only need a pair of formulas hard to interpolate, i.e. a pair of NP sets $U$ and $V$ hard to separate. The set $U$ will be closed upwards and will satisfy the monotonocity condition of Theorem 2.1, so that we can use the monotone feasible interpolation. This is essential as lower bounds for monotone circuits are known (Theorem 3.5) but not for general circuits. $\left(\begin{array}{c}m \\ 2\end{array}\right)$.

Denote the set of two-element subsets of $\{1, \ldots, m\}$ by the suggestive symbol

Definition 3.4 Let $m, \omega, \xi \geq 1$. The set Clique $m, \omega(x, y)$ is a set of the following clauses in the atoms $x_{i j},\{i, j\} \in\left(\begin{array}{c}m \\ 2\end{array}\right)$, and $y_{u i}, u=1, \ldots, \omega$ and $i=1, \ldots, m$ :

$$
\begin{aligned}
& \text { 1. } \bigvee_{i \leq m} y_{u i} \text {, all } u \leq \omega, \\
& \text { 2. } \neg y_{u i} \vee \neg y_{v i} \text {, all } u<v \leq \omega \text { and } i=1, \ldots, m \text {, } \\
& \text { 3. } \neg y_{u i} \vee \neg y_{v j} \vee x_{i j} \text {, all } u<v \leq \omega \text { and }\{i, j\} \in\left(\begin{array}{c}
m \\
2
\end{array}\right) \text {. }
\end{aligned}
$$

The set Color $_{m, \xi}(x, z)$ is the set of the following clauses in the atoms $x_{i j}$, $\{i, j\} \in\left(\begin{array}{c}m \\ 2\end{array}\right)$, and $z_{i a}, i=1, \ldots, m$ and $a=1, \ldots, \xi$ : 


$$
\begin{aligned}
& \text { 1. } \bigvee_{a \leq \xi} z_{i a} \text {, all } i \leq m \text {, } \\
& \text { 2. } \neg z_{i a} \vee \neg z_{i b} \text {, all } a<b \leq \xi \text { and } i \leq m \text {, } \\
& \text { 3. }] \neg z_{i a} \vee \neg z_{j a} \vee \neg x_{i j} \text {, all } a \leq \xi \text { and }\{i, j\} \in\left(\begin{array}{c}
m \\
2
\end{array}\right) \text {. }
\end{aligned}
$$

Truth assignments to atoms $x_{i j}$ can be identified with undirected graphs with the vertex set $[m]$. Truth assignments to $y_{u i}$ such that $C l i q u e_{m, \omega}(x, y)$ is satisfied can be identified with injective maps from the set $[\omega]$ onto a clique in the graph determined by $x$, and truth assignments to $z_{i a}$ such that $\operatorname{Color}_{m, \xi}(x, z)$ is satisfied can be identified with colorings of the graph by $\xi$ colors. The set

$$
U:=\left\{x \mid \exists y \text { Clique }_{m, \omega}(x, y)\right\}
$$

is the set of graphs on $[m]$ with a clique of size $\geq \omega$, while the set

$$
V:=\left\{x \mid \exists z \text { Color }_{m, \xi}(x, z)\right\}
$$

is the set of graphs on $[m]$ colorable by $\leq \xi$ colors. It is obvious that $U$ is closed upwards (and, in fact, $V$ downwards) and satisfies the monotonicity condition of Theorem 2.1. Moreover, the formula

$$
\bigwedge \text { Clique }_{m, \omega} \wedge \bigwedge \text { Color }_{m, \xi}
$$

is obviosuly unsatifiable if $\omega>\xi$.

We need to use the following well-known lower bound (we formulte it only for a particular set of parameters).

Theorem 3.5 (Alon-Boppana[1]) Let $m \geq 3$, and put $\xi:=m^{1 / 2}$ (rounded to the nearest integer) and $\omega:=\xi+1$. Then any monotone circuit separating the corresponding sets $U$ and $V$ must have the size at least $2^{\Omega\left(m^{1 / 4}\right)}$.

Now we are ready to state and prove the lower bound. Denote by $A_{m}(x, y)$ the formula $\bigwedge$ Clique $_{m, m^{1 / 2}+1}(x, y)$ and by $B_{m}(x, z)$ the formula $\bigwedge \operatorname{Color}_{m, m^{1 / 2}}(x, z)$. Hence $n=m(m+1) / 2, s=\left(m^{1 / 2}+1\right) \cdot m$ and $t=m \cdot m^{1 / 2}$. The total size of formulas $A_{m}$ and $B_{m}$, and also of formula $D_{A_{m}, B_{m}}$, is $m^{O(1)}$.

Theorem 3.6 (main) Let $m \geq 3$. Then any $O B D D$ refutation of $D_{A_{m}, B_{m}}(w, f)$ must have the size at least $2^{m^{1 / 5}}$.

\section{Proof :}

Let $\pi$ be any linear ordering of variables $x, y$ and $z$ of formulas $A_{m}$ and $B_{m}$ that is block consistent with $y<z<x$. Let $\sigma$ be any ordering of variables $w$ and $f$. Assume that $D_{A_{m}, B_{m}}$ has a size $S \sigma$-OBDD refutation.

By Lemma 3.3 then there is a $\pi$-OBDD refutation of $A_{m} \wedge B_{m}$ of size at most $S$. By Lemma 3.2 any such refutation yields a monotone ciruit separating $U$ from $V$ of size at most $S^{O(\log (n))}$.

By Theorem 3.5 then $S^{O(\log (n))} \geq 2^{\Omega\left(m^{1 / 4}\right)}$, i.e. $S \geq 2^{\Omega\left(m^{1 / 5}\right)}$.

q.e.d.

Acknowledgements: I thank A. Atserias (Barcelona) for discussions about $[2]$. 


\section{References}

[1] N. Alon, and R. Boppana, The monotone circuit complexity of Boolean functions, Combinatorica, 7(1), (1987), pp.1-22.

[2] A. Atserias, P. Kolaitis, and M. Vardi, Constraint propagation as a proof system, 10th Int.Conf. on Principles and Practice of Constraint Programing, LN in Computer Science vol.3258, Springer, (2004), pp.77-91.

[3] M. L. Bonet, T. Pitassi, and R. Raz, Lower bounds for cutting planes proofs with small coefficients, J. of Symbolic Logic,(1997), pp.708-728.

[4] R. E. Bryant, Graph-based algorithms for Boolean function manipulation, IEEE Transactions on Computing, C-35, (1986), pp.677-691.

[5] R. E. Bryant, Syntactic Boolean manipulation with ordered binary decision diagrams, ACM Computing Surveys, 2493), (1992), pp.293-318.

[6] S. A. Cook and A. R. Reckhow, The relative efficiency of propositional proof systems, J. Symbolic Logic,44(1), (1979), pp.36-50.

[7] R. Dechter, Constraint processing, Morgan and Kaufman, (2003).

[8] J. Krajíček, Lower bounds to the size of constant-depth propositional proofs, Journal of Symbolic Logic, 59(1), (1994), pp.73-86.

[9] J. Krajíček, Bounded arithmetic, propositional logic, and complexity theory, Encyclopedia of Mathematics and Its Applications, Vol. 60, Cambridge University Press, (1995).

[10] J. Krajíček, Interpolation theorems, lower bounds for proof systems, and independence results for bounded arithmetic, J. of Symbolic Logic, 62(2), (1997), pp.457-486.

[11] J. Krajíček, Propositional proof complexity I., lecture notes available at

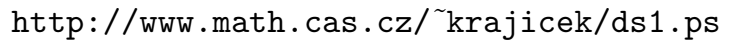

[12] J. Krajíček, and P. Pudlák, Some consequences of cryptographical conjectures for $S_{2}^{1}$ and EF", in: Logic and Computational Complexity (Proc. of the meeting held in Indianapolis, October 1994), Ed. D. Leivant, SpringerVerlag, Lecture Notes in Computer Science, Vol. 960, (1995), pp.210-220.

Revised version in: Information and Computation, Vol. 140 (1), (January 10, 1998), pp.82-94.

[13] E. Kushilevitz, and N. Nisan, Communication complexity, Cambridge University Press, (1996).

[14] P. Pudlák, Lower bounds for resolution and cutting plane proofs and monotone computations, J. of Symbolic Logic,(1997), pp.981-998. 
[15] P. Pudlák, The lengths of proofs, in: Handbook of Proof Theory, S.R.Buss ed., Elsevier, (1998), pp.547-637.

[16] A. A. Razborov, Unprovability of lower bounds on the circuit size in certain fragments of bounded arithmetic, Izvestiya of the R.A.N., 59(1), (1995), pp.201-224.

[17] I. Wegener, The complexity of Boolean functions, John Willey and Sons and Teubner Verlag, (1987).

[18] I. Wegener, Branching programs and binary decision diagrams - theory and applications, SIAM Monographs in Discrete Mathematics and Its Applications, (2000). 